\title{
The role of the Cronobacter sakazakii ProP C-terminal coiled coil domain in osmotolerance
}

\author{
Audrey Feeney ${ }^{\dagger}$, Christopher D Johnston $^{\dagger}$, Alan Lucid, Jim O’Mahony, Aidan Coffey, Brigid Lucey \\ and Roy D Sleator
}

\begin{abstract}
Background: We investigate the role of the C-terminal coiled coil of the secondary proline porter ProP in contributing to Cronobacter sakazakii osmotolerance.

Findings: The extended C-terminal domain of ProP1 (encoded by ESA_02131) was spliced onto the truncated C-terminal end of ProP2 (encoded by ESA_01706); creating a chimeric protein (ProPc) which exhibits increased osmotolerance relative to the wild type.
\end{abstract}

Conclusions: It appears that the C-terminal coiled coil domain tunes ProP at low osmolality, whereas ProP transporters lacking the coiled coil domain are more active at a higher osmolality range.

\section{Introduction}

Survival of the foodbourne pathogen Cronobacter sakazakii in low water activity $\left(\mathrm{a}_{\mathrm{w}}\right)$ environments, e.g. powdered infant formula (PIF), is largely attributed to the accumulation of organic compounds termed osmolytes or compatible solutes [1,2]. Synthesised de novo, or transported from the bathing solution[3], compatible solutes function to increase cell turgor thereby counterbalancing the external osmotic upshift and preventing water loss from the cell, which if left unchecked can lead to plasmolysis and ultimately cell death [4].

In Escherichia coli, a model organism for the study of bacterial osmoadaptation, the transmembrane protein ProP is perhaps the best characterised compatible solute uptake system; facilitating the uptake of both proline and glycine betaine [5]. A member of the major facilitator superfamily (MFS), E. coli ProP is a 500 -amino acid protein comprising of 12 transmembrane domains and a characteristic carboxy-terminal extension [5,6]. In a previous in silico study we identified seven ProP homologues on the C. sakazakii BAA-894 genome; one of which, ESA_02131, encodes a protein exhibiting 90\% identity to E. coli ProP [2]. While the remaining six homologues encode proteins exhibiting features of classic

\footnotetext{
*Correspondence: roy.sleator@cit.ie

${ }^{\dagger}$ Equal contributors

Department of Biological Sciences, Cork Institute of Technology, Rossa Avenue, Bishopstown, Cork, Ireland
}

secondary transporters, they are all 60-70 amino acids shorter than the E. coli ProP; lacking the extended carboxyl tail [2]. Notwithstanding the lack of structural consistency, particularly at the C-terminal end, we have shown that six of the seven C. sakazakii proP homologues contribute to C. sakazakii osmotolerance, albeit to varying degrees [7].

Culham et al. [5] first described the E. coli ProP as harbouring unusual structural features which appeared unique within the transporter superfamily. This study predicted the formation of an alpha helical coiled coil resulting from the presence of the carboxyl terminal extension [5]. Indeed, a synthetic polypeptide corresponding to the C-terminal extension of ProP formed a dimeric alpha helical coiled coil [6]. Interestingly, when amino acid changes were introduced to the coiled coil, ProP required a larger osmotic upshift to become activated [6], suggesting that the C-terminal domain likely plays a role in osmosensing. Furthermore, a derivative of ProP which lacked the 26 amino acid C-terminal domain was expressed, but inactive [6]. In contrast, despite the structural degeneracy observed between the homologues, C. sakazakii ProP homologues lacking the $\mathrm{C}$-terminal extension do contribute to osmotolerance, albeit to a lesser extent than the extended ProP (which we designate Prop1) encoded by ESA_02131 [7].

While several studies have focused on elucidating the role of the carboxyl extension in E. coli $[5,6,8]$, little is 
known about the role, if any, of the ProP1 carboxyl extension in the far more osmotolerant C. sakzakii. Herein, we investigate the role of the $\mathrm{C}$-terminal coiled coil of ProP1 in contributing to C. sakazakii osmotolerance, by creating a chimeric protein (ProPc) in which the extended C-terminal domain of ProP1 (encoded by ESA_02131) is spliced onto the truncated C-terminal end of ProP2 (encoded by ESA_01706).

\section{Material and methods}

\section{Bacterial strains and growth conditions}

Bacterial strains and plasmids used in this study are listed in Table 1.

\section{Creation of the chimeric ProPc protein}

PCR primers (Table 2) were designed for each proP homologue based on C. sakazakii strain BAA-894 sequence data available from the NCBI database (NC_009778.1). The formation of the chimeric ProP protein (ProPc), which consists of the extended coiled coil region of ProP1 (amino acid position 422 to 505) fused to the C-terminus of ProP2 (encoded by ESA_01706), was performed using a modified SOEing (Splicing by overlap extension) technique [12]. In silico comparative analysis of the native ProP1 and ProP2 sequences, revealed a point of amino acid homology within the twelfth predicted transmembrane domain, a leucine/isoleucine/threonine triplet (LIT) at position 422-424 and 437-439 respectively, which was selected as the splice site. Briefly, the fusion was performed using three separate PCR reactions: the first PCR (primer set Chimeric-01706) resulted in an ESA_01706 (proP2) amplicon lacking the C-terminal extension but containing a 15-bp 3'overhang corresponding to the LIT triplet of the ProP1 C- terminal extension. The second
PCR (primer set Chimeric-02131CTE) formed an amplicon of 210-bp encoding ProP1 C-terminal extension with a 5'-overhang, also corresponding to the LIT triplet. The final PCR (primer set Chimeric-01706-F Chimeric02131tail-R) was performed with the two previous amplicons as template; resulting in a final product of 1,623-bp, representing the ESA_01706 native promoter and modified coding region (encoding the fused ProP1 C-terminal extension after the LIT triplet). This product was digested with restriction enzymes BamHI and HindIII and ligated to a similarly digested pUC18 vector forming pUC18: ESA_01706CTE (C-Termini Extension). The integrity of the chimeric sequence was confirmed by sequencing (MWG Operon, Germany and GATC, Germany) and transformed into E. coli MKH13.

\section{Osmotolerance assay}

Overnight cultures of E. coli MKH13 clones expressing the wild-type and chimeric ProP proteins (ProP1, ProP2 and ProPC respectively) were grown at $37^{\circ} \mathrm{C}$ with shaking at $200 \mathrm{rpm}$ in either $10 \mathrm{ml} \mathrm{LB}$ or M9 minimal media containing $0.5 \%$ glucose, $0.04 \%$ arginine, $0.04 \%$ isoleucine, $0.04 \%$ valine (Sigma-Aldrich Co.). Cells were pelleted by centrifugation at 5,000 g, washed and re-suspended in $200 \mu \mathrm{l} 1 / 4$ strength Ringer's solution. The cell suspension was added to the appropriate filter sterilized media with varying concentrations (0-10\%) of added $\mathrm{NaCl}$. Growth was monitored in the relevant media over a 48 hour period, with optical density $\left(\mathrm{OD}_{600}\right)$ readings being taken every hour. Triplicate readings were taken and graphs were plotted using SigmaPlot version 11.0. E. coli MKH13 harbouring the empty pUC18 plasmid was used as a negative control.

Table 1 Bacterial strains and plasmids

\begin{tabular}{|c|c|c|}
\hline Strain or plasmid & Relevant genotype or characteristics & $\begin{array}{l}\text { Source or } \\
\text { reference }\end{array}$ \\
\hline \multicolumn{3}{|l|}{ Plasmids } \\
\hline pUC18 & Amp', lacZ', pMB9 replicon & [9] \\
\hline pUC18: ESA_02131 & pUC18 harboring ESA_02131 gene under control of the native promoter & [7] \\
\hline pUC18: ESA_01706 & pUC18 harboring ESA_01706 gene under control the native promoter & [7] \\
\hline pUC18: ESA_01706CTE & $\begin{array}{l}\text { pUC18 harboring chimeric ESA_01706 with fused C-terminal extension (ESA_02131) under } \\
\text { control of the native promoter }\end{array}$ & This work \\
\hline \multicolumn{3}{|l|}{ Strains } \\
\hline Cronobacter sakazakii BAA-894 & C.sakazakii strain isolated from powdered formula associated with neonatal intensive care unit & [10] \\
\hline Escherichia coli DH5a & Intermediate cloning host.supE44 $\Delta$ lacU169(80lacZAM15)R17 recA1 endA1 gyrA96 thi-1 relA1 & Invitrogen \\
\hline MKH13 & 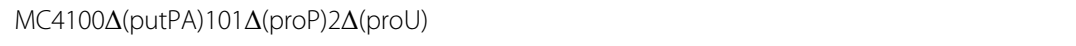 & [11] \\
\hline MKH13 pUC18:ESA_02131+ & Host strain harbouring pUC18: ESA_02131 plasmid. Ampr & [7] \\
\hline MKH13 pUC18:ESA_01706+ & Host strain harbouring pUC18: ESA_01706 plasmid. Ampr & [7] \\
\hline MKH13 pUC18:ESA_01706CTE & Host strain harbouring pUC18: ESA_01706CTE plasmid. Amp ${ }^{r}$ & This work \\
\hline
\end{tabular}


Table 2 Primers

\begin{tabular}{|c|c|c|c|c|}
\hline Primer name & & Primer sequence (5' to $\left.3^{\prime}\right)$ & Length & Characteristics \\
\hline \multirow[t]{2}{*}{ ESA_02131 } & $\mathrm{F}$ & CATCGGCCGACAGGCCAGTCAATGAATGATGC & 32 & Eagl cut site \\
\hline & $\mathrm{R}$ & CATTCTAGAGAGTACAACGGAATGCGGGG & 29 & Xbal cut site \\
\hline \multirow[t]{2}{*}{ ESA_01706 } & $\mathrm{F}$ & CATTCTAGAGTCGGGCGGCTCTTIATCTGG & 30 & Xbal cut site \\
\hline & $\mathrm{R}$ & CATGGATCCTTGACCAGATGACGCAGTCTTTC & 32 & BamHI cut site \\
\hline \multirow[t]{2}{*}{ Chimeric-01706 } & $\mathrm{F}$ & AATAAGCTTGTGGCTIITTATGCCGGGCTGC & 31 & Hindlll cut site \\
\hline & $\mathrm{R}$ & CAGGCCAGTAATCAGCGCCGCGCCCATGAC & 30 & 3' SOEing overhang \\
\hline \multirow[t]{2}{*}{ Chimeric-02131CTE } & $\mathrm{F}$ & CGCGGCGCTGATTACTGGCCTGACGATGAAAG & 32 & 5' SOEing overhang \\
\hline & $\mathrm{R}$ & AATGGATCCTTACTCGTTAATACGAGGATGCTGG & 34 & BamHI cut site \\
\hline \multirow[t]{2}{*}{ pUC18 MCS Check } & $\mathrm{F}$ & CATTAGCTCACTCATTAGGCACC & 23 & pUC18 insert check \\
\hline & $\mathrm{R}$ & CATTGTAAAACGACGGCCAGTG & 22 & pUC18 insert check \\
\hline
\end{tabular}

\section{Results}

\section{C. sakazakii ProP structures}

Based on sequence similarity to the E. coli ProP protein, we identified ProP1 (the product of ESA_02131) as the most likely ProP homolog in C. sakazakii; exhibiting 90\% amino acid sequence identity and structural features characteristic of $E$. coli ProP. Indeed, further analysis using TMHMM and TexTopo software predicted ProP1 to be a membrane protein with 12-transmembrane domains, an extended central hydrophilic loop and carboxy terminal extension (Figure 1). While the remaining five ProP homologues on the C. sakazakii BAA-894 genome were also predicted to encode proteins with 12 transmembrane domains and an extended central hydrophilic loop, they each lacked the extended carboxy-terminal domain identified in ProP1, a feature which likely affects the final protein structure and function.

Figure 1B illustrates the tertiary structure for ProP1 (predicted using the I-TASSER server $[13,14]$ ). Most notably the presence of a coiled coil domain is evident as a result of the extended carboxy-terminal identified by sequence analysis. The coiled coil domain likely protrudes into the intracellular cytoplasm of the organism where its function remains unclear. By contrast, the tertiary structure of ProP2 (Figure 1A), representative of the remaining 6 ProP homologues and exhibiting 40\% identity to E. coli ProP and $49 \%$ identity to ProP1, lacks the coiled coil domain at the carboxy-terminal end.

\section{Chimeric protein (ProPc) expression in E. coli MKH13}

The osmoprotective properties of ProP1, ProP2 and ProPc were measured and compared in E. coli MKH13; an osmosensitive mutant incapable of growth in high osmolality environments $(\geq 4 \%)$. The pUC18 plasmid containing each gene of interest was transformed to $E$. coli MKH13. Transformation efficiencies of $60 \mathrm{CFU} / \mu \mathrm{g}$ DNA were achieved, with successful transformation being confirmed by colony PCR, followed by sequencing. Transformants were screened for osmotolerance on media (both
LB and M9 plus $1 \mathrm{mM}$ proline) containing between $4 \%$ and $10 \%$ added $\mathrm{NaCl}$.

\section{Assessment of osmotolerance}

To determine the effect of each ProP homologues, both native (ProP1 and ProP2) and chimeric (ProPc), on the osmotolerance of $E$. coli MKH13, each of the strains was grown in media containing varying concentrations of $\mathrm{NaCl}$. Growth was monitored over a 48 hour period in minimal media supplemented with $1 \mathrm{mM}$ proline and containing $0-10 \%$ added $\mathrm{NaCl}$.

Media containing $5 \% \mathrm{NaCl}$ yielded the most discriminatory results. While E. coli MKH13 expressing the empty pUC18 vector showed no growth at $5 \%$, each of the other three strains tested conferred some degree of osmotolerance on the host (Figure 2A). The strain expressing ProP1 was the most osmotolerant, with a maximum optical density $\left(\mathrm{OD}_{600}\right)$ of 0.326 after 37 hours growth at $5 \%$ $\mathrm{NaCl}$. The strain possessing ProPc grew to an OD significantly higher than the strains expressing either ProP1 or ProP2. E. coli MKH13 expressing ProP2 in $5 \% \mathrm{NaCl}$ grew to a maximum OD of 0.111 after 48 hours, whereas E. coli MKH13 containing ProPc grew to a final OD of 0.189 at the same time point. Interestingly, growth of the stains expressing ProP2 and the chimeric protein continued to increase up to 48 hours, while growth of $E$. coli MKH13 expressing ProP1 reached maximum OD after only 37 hours.

Each E. coli MKH13 strain expressing a proP gene of interest conferred osmotolerance (Figure 2B). As expected, E. coli MKH13 demonstrated a significant reduction in growth rate as $\mathrm{NaCl}$ concentrations increased, with a final growth rate of $0.0004 \mathrm{hr}^{-1}$ recorded in media supplemented with $4 \% \mathrm{NaCl}$ and no subsequent growth recorded thereafter. E. coli MKH13 expressing ProP1 demonstrated the highest osmotolerance of all the strains tested with growth rates of $0.009 \mathrm{hr}^{-1}$ to $0.004 \mathrm{hr}^{-1} \mathrm{re}-$ corded in media supplemented with $5 \%$ to $10 \% \mathrm{NaCl}$ respectively. The next most osmotolerant strain was that 


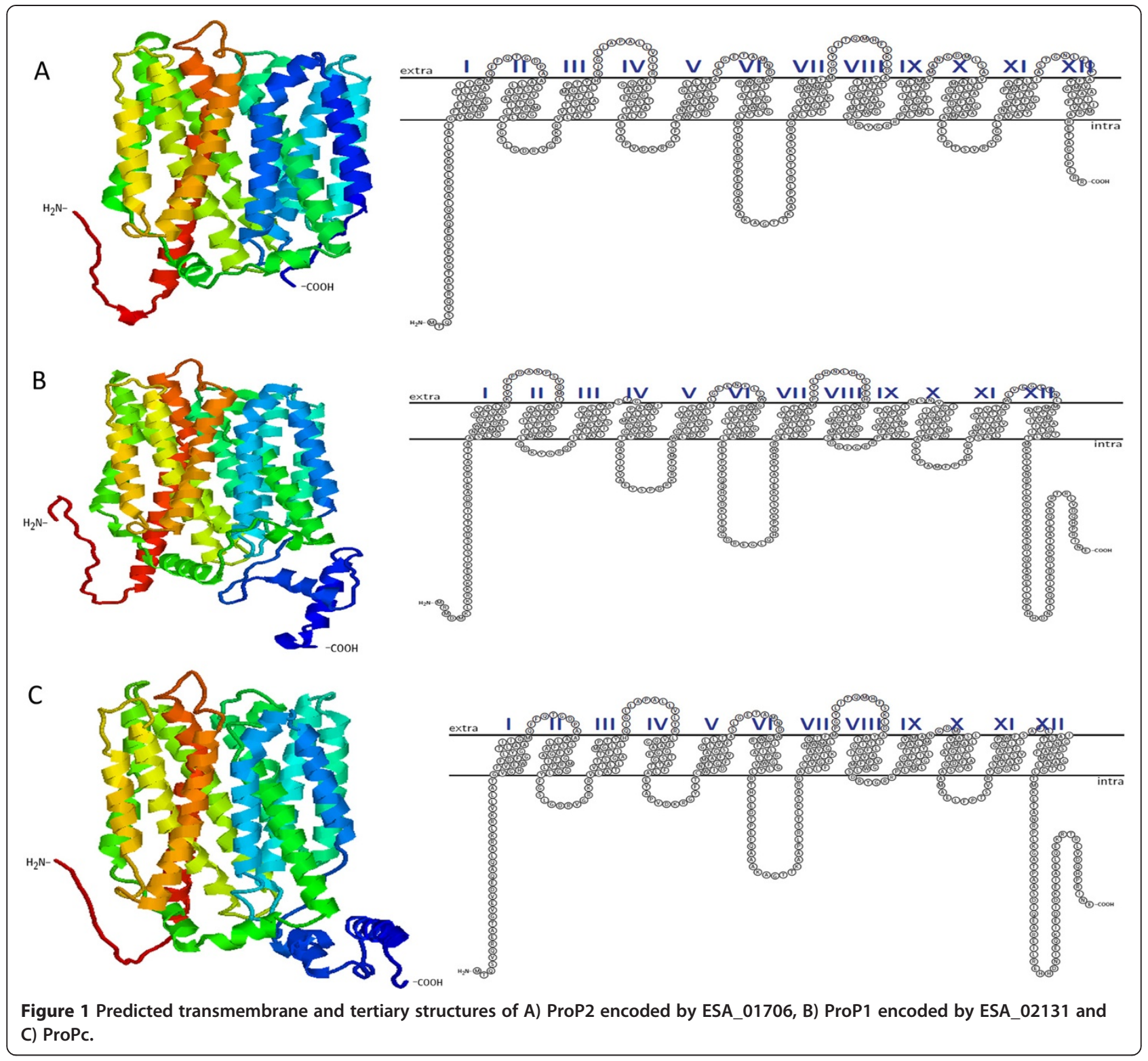

expressing ProPc. Growth rates of $0.004 \mathrm{hr}^{-1}$ to $0.003 \mathrm{hr}^{-1}$ were recorded in media supplemented with $5 \%$ to $10 \%$ $\mathrm{NaCl}$ respectively (Table 3 ). This was higher than the growth rates observed when E. coli MKH13 expressing the native ESA_01706 gene (ProP2) was grown in a high osmolality environment, suggesting an important role for the C. sakazakii ProP C-terminal extension in osmotolerance.

\section{Discussion}

A unique feature of the neonatal pathogen C. sakazakii is its ability to survive for prolonged periods in environments of low $\mathrm{a}_{\mathrm{W}}$, such as powdered infant formula (PIF), making it a significant cause for concern [15]. Indeed, up to $80 \%$ of infants infected with C. sakazakii die within days of birth, while survivors often suffer delayed neurological symptoms, brain abscesses or hydrocephalus $[16,17]$. However, despite this, little is known about the molecular mechanisms that allow this organism to survive in environments such as PIF where it is subject to extreme hyper-osmotic stress.

In a previous in silico study we identified seven copies of an E. coli proP homolog on the BAA-894 genome. Physiological analysis confirmed that six of the proP homologues identified played a role in osmotolerance. The availability of osmolytes in the media also had an effect on the osmotolerance of the host, with growth rates varying depending on the type or variety of compatible solutes present [7]. While all six ProP proteins exhibited features characteristic of classic secondary transporters, 


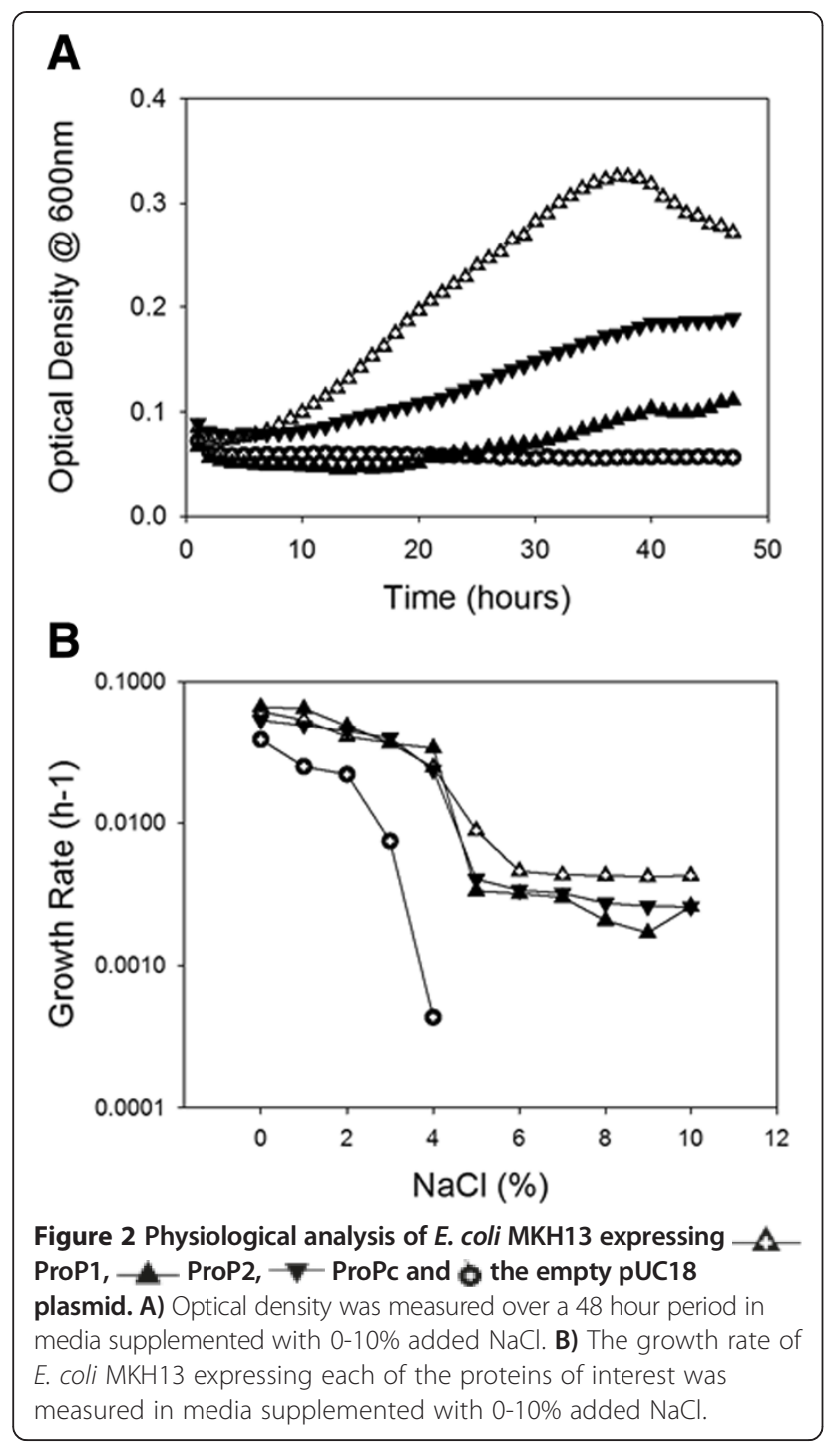

five of the proteins were between 60-70 amino acids shorter than ProP1; lacking the characteristic C-terminal cytoplasmic extension Previous studies in our lab have demonstrated that the six C. sakazakii ProP homologues lacking the $\mathrm{C}$-terminal coiled coil are significantly less osmoprotective than ProP1 [7], suggesting an important role for this domain in modulating $C$. sakazakii osmotolerance.
In the current study, the C. sakazakii ProP2 (encoded by ESA_01706) was chosen as the prototypical ProP homologue to study the role of the alpha helical coiled coil in osmotolerance. Genetic splicing yielded a chimeric protein structure (ProPc) possessing the native ProP2 domains in addition to the C-terminal alpha helical coiled coil domain from ProP1 (Figure 1C). E. coli MKH13 expressing ProPc grew to a higher OD in minimal media supplemented with proline, when compared to the native protein ProP2 which lacked the extended coiled coil domain (Figure 2A). These data demonstrate that the addition of the coiled coil domain from ProP1 to ProP2 results in a protein with an increased osmoprotective effect on the usually osmotically sensitive E. coli MKH13. However, as the osmolality of the medium increased, this trend appeared to reverse with OD readings becoming similar at $9 \% \mathrm{NaCl}$ and the chimeric protein growing to a higher OD than the native in $10 \% \mathrm{NaCl}$, suggesting that the extent of osmotic pressure also has a role to play in the activity of the proteins.

The role of the C-terminal domain of other osmolyte transporters, such as BetP (Corynebacterium glutamicum) and OpuA (Bacillus subtilis), was demonstrated to be important for the activation of these proteins during an increase in the osmolality of the surrounding medium [18]. Furthermore, Culham et al. created a synthetic polypeptide corresponding to the $\mathrm{C}$-terminal domain of $E$. coli ProP which formed a dimeric alpha helical coiled coil structure [6], similar to the coiled coil structure of ProP1 (illustrated in Figure 1A). In the same study ProP proteins from both E. coli and Agrobacterium tumefaciens, possessing the characteristic alpha helical coiled coil, were activated at a lower osmolality than orthologues lacking the coiled coil structure. C. glutamicum possesses a ProP protein which lacks the C-terminal alpha helical coiled coil domain and, presumably as a result of this, requires a higher osmolality for activation [6]. E. coli ProP variants lacking the coiled coil or with an amino acid substitution disrupting the formation of the alpha helical coiled coil, also require a larger osmotic upshift than the wild-type transporter [6,19]. This study demonstrates that the activity of these ProP orthologues is dependent on the osmolality of the surrounding medium, and the alpha helical coiled coil is believed to tune the transporter to

Table 3 Growth rate and optical density @ 600 nm

\begin{tabular}{|c|c|c|c|c|c|c|c|c|c|c|c|c|c|c|}
\hline \multirow{2}{*}{$\begin{array}{l}\text { Protein } \\
\text { expressed }\end{array}$} & \multirow{2}{*}{$\begin{array}{l}\text { Gene locus } \\
\text { tags }\end{array}$} & \multirow[t]{2}{*}{ Name } & \multicolumn{2}{|l|}{$5 \%$} & \multicolumn{2}{|l|}{$6 \%$} & \multicolumn{2}{|l|}{$7 \%$} & \multicolumn{2}{|l|}{$8 \%$} & \multicolumn{2}{|l|}{$9 \%$} & \multicolumn{2}{|l|}{$10 \%$} \\
\hline & & & $\begin{array}{l}\text { Max. } \\
O D\end{array}$ & $\begin{array}{l}\text { Growth } \\
\text { rate }\left(h r^{-1}\right)\end{array}$ & $\begin{array}{l}\text { Max. } \\
O D\end{array}$ & $\begin{array}{l}\text { Growth } \\
\text { rate }\left(h r^{-1}\right)\end{array}$ & $\begin{array}{l}\text { Max. } \\
O D\end{array}$ & $\begin{array}{l}\text { Growth } \\
\text { rate }\left(h r^{-1}\right)\end{array}$ & $\begin{array}{l}\text { Max. } \\
O D\end{array}$ & $\begin{array}{l}\text { Growth } \\
\text { rate }\left(h r^{-1}\right)\end{array}$ & $\begin{array}{l}\text { Max. } \\
O D\end{array}$ & $\begin{array}{l}\text { Growth } \\
\text { rate }\left(h r^{-1}\right)\end{array}$ & $\begin{array}{l}\text { Max. } \\
O D\end{array}$ & $\begin{array}{l}\text { Growth } \\
\text { rate }\left(h r^{-1}\right)\end{array}$ \\
\hline Native & ESA_02131 & ProP1 & 0.326 & 0.009 & 0.144 & 0.005 & 0.152 & 0.004 & 0.177 & 0.004 & 0.181 & 0.004 & 0.204 & 0.004 \\
\hline Native & ESA_01706 & ProP2 & 0.111 & 0.003 & 0.096 & 0.002 & 0.083 & 0.002 & 0.074 & 0.002 & 0.127 & 0.002 & 0.171 & 0.002 \\
\hline Chimeric & $\begin{array}{l}\text { ESA_02131 } \\
\text { ESA_01706 }\end{array}$ & ProPc & 0.189 & 0.004 & 0.130 & 0.003 & 0.113 & 0.003 & 0.117 & 0.003 & 0.124 & 0.003 & 0.141 & 0.003 \\
\hline
\end{tabular}


osmoregulate the cell over a low osmolality range [19]. These data may therefore offer an explanation for the increased growth observed in E. coli MKH13 expressing ProP2, which lacks the coiled coil domain, in media supplemented with $10 \% \mathrm{NaCl}$ relative to either ProP1 or ProPc (Figure 2). It is likely that the coiled coil domain of C. sakazakii ProP1 has a similar tuning function. Furthermore, the presence of multiple ProP porters lacking the $\mathrm{C}$-terminal coiled coil domain, and therefore only active at a higher osmolality, may well explain the extreme osmotolerance unique to C. sakazakii; allowing the pathogen to survive in environments like PIF. The ProP1 protein, on the other hand possessing the coiled coil, may be the only osmolyte transporter required to respond to low or moderate hyperosmotic challenge.

\section{Conclusion}

The addition of the coiled coil domain from ProP1 to ProP2 resulted in a chimeric protein (ProPc) which demonstrated higher osmotolerance compared to the native ProP2 (under moderate osmotic stress conditions). Furthermore, the growth rate of E. coli MKH13 expressing ProP2 increased in minimal media supplemented with $10 \% \mathrm{NaCl}$; suggesting that, as is the case in E. coli [19], the coiled coil domain tunes ProP at low osmolality, whereas ProP transporters lacking the coiled coil domain are more active at a higher osmolality range.

\section{Competing interests}

The authors declare that they have no competing interests.

\section{Authors' contributions}

$A F, C J$ and $A L$ carried out the the experimental work. AF drafted the manuscript together with together with $\mathrm{CJ}, \mathrm{AL}$ and RDS. All authors read and approved the final manuscript.

\section{Acknowledgements}

RDS is Coordinator of the EU FP7 ClouDx-i project (grant number 324365). AF is funded by an IRCSET EMBARK Postgraduate Scholarship RS/2010/2300, AL is funded by an IRC fellowship (RS/2012/219), CJ is funded by the Department of Agriculture under the Food Institutional Research Measure (08RDCIT617).

Received: 29 October 2014 Accepted: 25 November 2014

Published online: 16 December 2014

\section{References}

1. Yancey PH, Clark ME, Hand SC, Bowlus RD, Somero GN: Living with water stress: evolution of osmolyte systems. Science 1982, 217(4566):1214-1222.

2. Feeney A, Sleator RD: An in silico analysis of osmotolerance in the emerging gastrointestinal pathogen Cronobacter sakazakii. Bioeng Bugs 2011, 2(5):260-270

3. Sleator RD, Gahan CG, Hill C: Identification and disruption of the proBA locus in Listeria monocytogenes: role of proline biosynthesis in salt tolerance and murine infection. Appl Environ Microbiol 2001, 67(6):2571-2577.

4. Sleator RD, Hill C: Bacterial osmoadaptation: the role of osmolytes in bacterial stress and virulence. FEMS Microbiol Rev 2002, 26(1):49-71.

5. Culham DE, Lasby B, Marangoni AG, Milner JL, Steer BA, Van Nues RW, Wood JM: Isolation and sequencing of escherichia coli gene proP reveals unusual structural features of the osmoregulatory proline/betaine transporter, ProP. J Mol Biol 1993, 229(1):268-276.
6. Culham DE, Tripet B, Racher KI, Voegele RT, Hodges RS, Wood JM: The role of the carboxyl terminal a-helical coiled-coil domain in osmosensing by transporter ProP of Escherichia coli. J Mol Recognit 2000, 13(5):309-322.

7. Feeney A, Johnston CD, Govender R, O'Mahony J, Coffey A, Sleator RD: Analysis of the role of the Cronobacter sakazakii ProP homologues in osmotolerance. Gut Pathogens 2014, 6:15.

8. Verheul A, Wouters JA, Rombouts FM, Abee T: A possible role of ProP, ProU and CaiT in osmoprotection of Escherichia coli by carnitine. J Appl Microbiol 1998, 85(6):1036-1046.

9. Vieira J, Messing J: The pUC plasmids, an M13mp7-derived system for insertion mutagenesis and sequencing with synthetic universal primers. Gene 1982, 19(3):259-268.

10. Kucerova E, Clifton SW, Xia X-Q, Long F, Porwollik S, Fulton L, Fronick C, Minx P, Kyung K, Warren W, Fulton R, Feng D, Wollam A, Shah N, Bhonagiri V, Nash WE, Hallsworth-Pepin K, Wilson RK, McClelland M, Forsythe SJ: Genome sequence of Cronobacter sakazakii BAA-894 and comparative genomic hybridization analysis with other Cronobacter species. PLoS One 2010, 5(3):e9556

11. Kempf B, Bremer E: OpuA, an osmotically regulated binding proteindependent transport system for the osmoprotectant glycine betaine in bacillus subtilis. J Biol Chem 1995, 270(28):16701-16713.

12. Sleator RD, Gahan CGM, O'Driscoll B, Hill C: Analysis of the role of betL in contributing to the growth and survival of Listeria monocytogenes LO28. Int J Food Microbiol 2000, 60(2-3):261-268.

13. Zhang Y: I-TASSER server for protein 3D structure prediction. BMC Bioinformatics 2008, 9(1):40.

14. Roy A, Kucukural A, Zhang Y: I-TASSER: a unified platform for automated protein structure and function prediction. Nat Protoc 2010, 5(4):725-738.

15. Feeney A, Sleator RD: The human gut microbiome: the ghost in the machine. Future Microbiol 2012, 7(11):1235-1237.

16. Townsend SM, Hurrell E, Gonzalez-Gomez I, Lowe J, Frye JG, Forsythe S, Badger JL: Enterobacter sakazakii invades brain capillary endothelial cells, persists in human macrophages influencing cytokine secretion and induces severe brain pathology in the neonatal rat. Microbiol 2007, 153(Pt 10):3538-3547.

17. Burdette JHSC: Enterobacter sakazakii brain abscess in the neonate: the importance of neuroradiologic imaging. Pediatric Radiology 2000 30(1):33-34

18. Keates RAB, Culham DE, Vernikovska YI, Zuiani AJ, Boggs JM, Wood JM: Transmembrane Helix I and Periplasmic Loop 1 of Escherichia coli ProP are involved in osmosensing and osmoprotectant transport. Biochemistry 2010, 49(41):8847-8856.

19. Tsatskis Y, Khambati J, Dobson M, Bogdanov M, Dowhan W, Wood JM: The osmotic activation of transporter ProP is tuned by both its c-terminal coiled-coil and osmotically induced changes in phospholipid composition. J Biol Chem 2005, 280(50):41387-41394.

doi:10.1186/s13099-014-0046-9

Cite this article as: Feeney et al.: The role of the Cronobacter sakazakii ProP C-terminal coiled coil domain in osmotolerance. Gut Pathogens 2014 6:46.

\section{Submit your next manuscript to BioMed Central and take full advantage of:}

- Convenient online submission

- Thorough peer review

- No space constraints or color figure charges

- Immediate publication on acceptance

- Inclusion in PubMed, CAS, Scopus and Google Scholar

- Research which is freely available for redistribution 